\title{
Factores críticos de la calidad del servicio de limpieza municipal asociados al riesgo de recolección de
} residuos peligrosos

\section{Critical factors of the quality of municipal cleaning service associated with the risk of hazardous waste} collection

Carlos Alberto Canelo Dávila ${ }^{{ }^{*}}$

\section{RESUMEN}

El objetivo fue describir los factores críticos de la percepción de la calidad del servicio de recolección de residuos peligrosos prestados por la municipalidad de Chachapoyas de Perú. Para ello se adaptó el modelo SERVPERF. La población estuvo conformada por los establecimientos generadores de residuos peligrosos ubicados en el ámbito urbano. Previo al análisis factorial se realizó un análisis de los datos ausentes y atípicos, se examinó la fiabilidad y correlación de la escala y se calculó la adecuación muestral de Kaiser-Meyer-Olkin. También se usó el método de análisis de componentes principales, la regla de Kaiser y se asumió el criterio de saturación. Se obtuvo una fiabilidad con el alfa de Cronbach de 0,65 y una prueba de Bartlett menor que 0,05. Los factores críticos para medir la percepción de la calidad fueron: seguridad (7,43\%), capacidad de respuesta $(6,32 \%)$ y confiabilidad $(6,26 \%)$. Por el contrario, los que menos lo explicaron fueron comunicación $(6,19 \%)$, credibilidad $(6,07 \%)$, profesionalismo $(5,91 \%)$ y participación ciudadana, que lograron medir sólo el $44,5 \%$ de su variabilidad original. La escala propuesta quedó constituida por tres factores: capacidad de respuesta con seis ítems, participación ciudadana con cinco ítems, y confiabilidad con cuatro ítems, que en total alcanzaron correlaciones mayores a 0,5 y explicaron el $69,71 \%$ de la varianza. Fue descartado el factor participación ciudadana así como los ítems P7, P9, P10, P12, P14, P15, P16 y P22 por presentar comunalidades menores que 0,60. En cambio los ítems P1, P2, P11, P18 y P21 alcanzaron valores factoriales por encima de 0,74 .

Palabras claves: Residuos sólidos, calidad de servicio, SERVPERF, percepción, análisis factorial.

\begin{abstract}
The goal was to describe the critical factors of the perception of the quality of the hazardous waste collection service provided by the municipality of Chachapoyas of Peru. For this the SERVPERF model was adapted. The population consisted of establishments that generate hazardous waste located in the urban area. Prior to the factor analysis, an analysis of the absent and atypical data was performed, the reliability and correlation of the scale was examined and the Kaiser-Meyer-Olkin sample adequacy was calculated. The principal component analysis method was also used, the Kaiser rule and the saturation criterion was assumed. Reliability was obtained with Cronbach's alpha of 0.65 and a Bartlett's test of less than 0.05 . The critical factors to measure the perception of quality were: safety $(7.43 \%)$, response capacity $(6.32 \%)$ and reliability $(6.26 \%)$. On the contrary, those who explained least were communication $(6.19 \%)$, credibility $(6.07 \%)$, professionalism $(5.91 \%)$ and citizen participation, which managed to measure only $44.5 \%$ of their original variability. The proposed scale was constituted by three factors: responsiveness with six items, citizen participation with five items, and reliability with four items, which in total reached correlations greater than 0.5 and explained $69.71 \%$ of the variance. The citizen participation factor was discarded as well as the items P7, P9, P10, P12, P14, P15, P16 and P22 for presenting communalities less than 0.60 . In contrast, items P1, P2, P11, P18 and $\mathrm{P} 21$ reached factorial values above 0.74 .
\end{abstract}

Keywords: Solid waste, service quality, SERVPERF, perception, factor analysis. 


\section{INTRODUCCIÓN}

El Listado Europeo de Residuos, la Convención de Basilea sobre el control de los movimientos transfronterizos de los desechos peligrosos y su eliminación, así como el Código de Regulación Federal de los Estados Unidos usan criterios distintos en las definiciones, identificación y clasificación de residuos peligrosos, por lo que cada país ha desarrollado sus regulaciones respecto al control de residuos peligrosos, de acuerdo a su realidad y necesidades (Orjuela-Yepes, 2013). En el Perú, la norma principal es la Ley de Gestión Integral de Residuos Sólidos (Poder Ejecutivo, 2016), e integra a aquellos que muestren por lo menos una de las siguientes características: autocombustibilidad, explosividad, corrosividad, reactividad, toxicidad, radioactividad o patogenicidad. El código penal peruano a través del delito de tráfico de residuos peligrosos sanciona esta infracción con pena privativa de la libertad no menor de cuatro años ni mayor de seis años. Por eso los gobiernos locales no deben realizar la recolección, tratamiento, transporte ni disposición final de residuos peligrosos. Sin embargo, existe un riesgo que esto se produzca con las consecuencias legales para los operadores y garantes administrativos de los servicios de limpieza, así como la generación de posibles daños ambientales y de salubridad en la comunidad.

El sector salud tiene a su cargo la fiscalización ambiental respecto al manejo de residuos sólidos en los establecimientos de salud y servicios médicos de apoyo públicos y privados (DIGESA, 2012), sin embargo el Organismo de Evaluación y Fiscalización Ambiental ha encontrado que el cumplimiento de esta función en los gobiernos subnacionales equivale en promedio nacional a 8.51 de un total de 20 puntos (OEFA, 2014), y respecto a los gobiernos locales provinciales ninguno cuenta con una escombrera autorizada. Así, sólo el 6\% tiene relleno sanitario autorizado y la fiscalización de rutas de transporte de residuos peligrosos en su jurisdicción únicamente es cumplida por el $2 \%$ (OEFA, 2016).

La calidad de un bien o servicio está compuesta por todos los atributos o propiedades que lo conforman y que le otorgan valor. La calidad de los productos puede medirse de manera sencilla pero se complica cuando se trata de la calidad de los servicios debido a sus características de intangibilidad, inseparabilidad, heterogeneidad y caducidad (Duque y Ramiro, 2012). La percepción de la calidad respecto a los servicios de limpieza municipal con el uso de instrumentos validados con análisis factorial está casi inexplorado por los generadores de residuos y poco estudiado por los ministerios competentes (ambiente, salud, transporte, agricultura, energía y producción), por lo que desarrollar escalas para su medición puede permitirnos contar con información para un mejor manejo y gestión de los residuos peligrosos domiciliarios y no domiciliarios, debido a los potenciales daños a los operadores municipales, la salud pública y el ambiente que se asocian con los riesgos en la prestación del servicio de limpieza municipal.

El objetivo de este estudio es describir los factores críticos de la calidad del servicio municipal de recolección de residuos asociados al riesgo de recolección de residuos peligrosos, lo que nos ayudará en el desarrollo de investigaciones aplicativas.

\section{MATERIALES Y MÉTODOS}

Para medir la calidad del servicio de recolección de residuos percibida por el usuario se empleó la escala proporcionada por Sáenz (2011) que estuvo basada en el modelo SERVPERF. El instrumento fue adaptado para los residuos peligrosos y tuvo siete dimensiones (Tabla 1-Apéndice A), que se correspondieron con los factores críticos tales como capacidad de respuesta, confiabilidad, profesionalismo del personal recolector, credibilidad, comunicación, participación ciudadana y seguridad. Esta última buscó medir la calidad del servicio imputable al usuario, quien fue considerado de vital importancia en la colaboración de la mejora continua del servicio de limpieza municipal, al supuestamente incidir de forma directa con su intervención en la calidad del mismo.

Como el número de ítems por dimensión interactúa 
con el tamaño de las comunalidades de los ítems y con el tamaño de la muestra, para la determinación de los ítems se consideró correcto no emplear ítems redundantes. Si bien es común en la práctica seleccionar si es posible tres ítems por factor como mínimo para obtener una solución factorial estable sobre todo cuando donde se dispone de un mínimo de 200 casos (Fabrigar et al., 1999; Ferrando y Anguiano-Carrasco, 2010), el instrumento propuesto se basa en la disponibilidad de establecimientos a encuestar.

El instrumento utilizó la escala Likert con cinco alternativas, asociando a cada respuesta una puntuación con la intención de transformar una apreciación cualitativa del ciudadano en una cuantitativa.

Las opciones con las que el usuario contó para responder variaron desde estar fuertemente en desacuerdo, muy en desacuerdo, incluyendo una posición neutral, hasta el otro extremo donde el entrevistado pudo expresar estar muy de acuerdo o fuertemente de acuerdo con las preguntas planteadas. Una respuesta de valor uno fue indicativo de una percepción de baja calidad del servicio en el ítem preguntado; mientras que una puntuación de valor cinco expresó una percepción de alta calidad del servicio. Esto fue válido para los ítems redactados positivamente. Para los ítems redactados de forma negativa se recodificaron las respuestas de forma tal que tuvieran el mismo sentido que los ítems positivos a la hora de cuantificar la calidad por dimensiones.

La población objeto de estudio estuvo conformada por los establecimientos generadores de residuos peligrosos ubicados en el ámbito urbano de la ciudad de Chachapoyas de Perú, en los cuales se realiza el servicio regular de recolección de residuos sólidos comerciales, quedando excluidas las viviendas.

El tamaño de la muestra se obtuvo con la siguiente formula:

$$
n=\frac{\left.Z-(1-\alpha / 2)^{2} \times N \sigma^{2}\right)}{e^{2} \times(N-1)+Z-(1-\alpha / 2)^{2} \times \sigma^{2}}
$$

Donde

$\mathrm{n}=$ muestra

$\mathrm{N}=375$ establecimientos

$Z-\alpha / 2=$ nivel de confianza de $95 \%$

$\sigma=$ desviación estándar de 0,05

$\mathrm{e}=$ error permisible de 0,061

A la muestra se adicionó un $20 \%$ más de establecimientos en caso de no poder realizar el trabajo de campo, obteniéndose 183 en total, los cuales fueron distribuidos según la zonificación de la ciudad en cuatro subsectores, realizando en cada uno de ellos el empadronamiento y entrevista a cada persona mayor de edad encargada del manejo de residuos, en horario diurno y en un periodo de seis días consecutivos.

Se partió del supuesto del análisis factorial clásico que los ítems se relacionan linealmente con los factores que miden, que las relaciones entre ellos también son lineales y que no son variables continuas, sino ordinales y discretas, por ser politómicas (tipo Likert), por lo que su distribución se consideró aproximadamente normal. Para la formulación del modelo y obtención de la matriz de datos se realizó un análisis factorial de tipo exploratorio (AFE) con la finalidad de estudiar la estructura de correlación entre los ítems, asumiendo que la asociación entre estos puede ser explicada por uno o más factores subyacentes, pues la variabilidad de cada ítem se produce por su comunalidad (a partir de la varianza común entre ese ítem y el resto de ítems que miden ese mismo factor común) y por su varianza no común (unicidad), el cual se considera como error (Lloret-Segura et al., 2014).

Previo al análisis factorial se realizó un análisis de los datos ausentes con la finalidad que los datos perdidos de los ítems no supere el 5\% y que el porcentaje de encuestados con datos perdidos fuera aceptable. Se revisó la existencia de datos atípicos o marginales con el propósito de buscar la existencia de valores extremos en un ítem o en una combinación de ellos, y evitar distorsiones en los resultados, para ello se empleó la media. Para la verificación del supuesto de normalidad 
de los ítems se empleó la prueba de contraste de bondad de ajuste Kolmogorov-Smirnov por ser muy sensible a pequeñas desviaciones de la normalidad en las muestras. La evaluación del supuesto de linealidad se evaluó examinando visualmente los diagramas matriciales de dispersión y cuando los puntos se organizaron a lo largo de una línea recta, se consideró que se cumplía con el mencionado supuesto. El diagnóstico de multicolinealidad entre los ítems se realizó con el objeto de identificar correlaciones elevadas o redundantes y evitar una solución factorial poco estable. Para ello se observó la matriz de correlación atendiendo a la existencia de valores iguales o superiores a 0.90. Para el análisis de fiabilidad de la consistencia interna de la escala se empleó el coeficiente Alpha de Cronbach (Oviedo y Campo-Arias, 2005).

Después de seleccionar la base de datos, la fase siguiente en el AFE fue calcular la matriz de correlación entre las variables relevantes del conjunto de datos. Se empleó una matriz tipo-R con la finalidad de comprobar si la correlación entre las variables analizadas es lo suficientemente grande como para justificar la factorización de la matriz de coeficientes de correlación, para ello una vez formulado el modelo y obtenida la matriz de datos el siguiente paso fue realizar el examen de la matriz de correlaciones con la finalidad de comprobar la existencia de agrupamientos relevantes entre los ítems y que los ítems que tuvieran correlación muy alta entre sí la tuvieran con el mismo factor o factores.

El Test de esfericidad de Bartlett permitió evaluar la hipótesis nula que afirma que las variables no están correlacionadas. Si los resultados obtenidos de dicha comparación resultaron con una alta correlación a un nivel de confiabilidad de $\mathrm{p}<0,05$, se debía rechazar la hipótesis nula y se consideró que las variables estaban lo suficientemente intercorrelacionadas para realizar el AFE. La matriz a factorizar se evaluó a través del cálculo de la medida de adecuación muestral de Kaiser-Meyer-Olkin (KMO) de Kaiser (1970) que refleja cuán grande son las correlaciones entre los ítems, tamaño de la muestra, número de factores y número de ítems. Se interpretó de manera semejante a los coeficientes de confiabilidad, huelga decir, con un rango de 0 a 1 ; es decir que si $K M O \geq 0,75$, la idea de realizar un análisis factorial es buena, pues sugiere una interrelación satisfactoria entre los ítems, si 0,75> $\mathrm{KMO} \geq 0,5$ la idea es aceptable y si $\mathrm{KMO}<0,5$ es inaceptable porque las correlaciones entre pares de ítems no pueden ser explicadas por los otros ítems, y por lo tanto, no es factible llevar a cabo el análisis factorial.

La extracción de factores se realizó con el método de análisis de componentes principales (Lloret-Segura et al., 2014) y para la determinación del número de factores se empleó la regla de Kaiser de extracción de factores con valores propios mayores a 1 . El otro criterio fue la aplicación del porcentaje de varianza explicada con la finalidad de analizar el porcentaje acumulado de la varianza total extraída con la extracción de factores de hasta $60 \%$ de la varianza total (Zamora et al., 2009). Para la rotación de factores y asignación de ítems, se asumió el criterio de que la saturación de un ítem nunca debió estar por debajo de 0,40 . Los ítems que no superaron ese valor debieron ser sometidos a un examen desde el punto de vista sustantivo y metodológico para decidir entre tres opciones: eliminar el ítem, revisar el ítem o añadir nuevos ítems que mostraran la faceta relacionada con ese ítem en particular.

\section{RESULTADOS Y DISCUSIÓN}

En la Tabla 2 (Apéndice A) se muestra el análisis de la matriz de correlaciones donde las dimensiones presentan factores comunes con distintos valores de correlación.

El índice Alfa de Cronbach de los 23 ítems de la escala inicial tiene un valor de 0,6 , y si bien es ideal obtener valores entre 0,70 y 0,90 , en ciencias sociales un valor mayor a 0,60 es considerado como de fiabilidad aceptable, lo que equivale a mencionar que la escala mide consistentemente la muestra de la población, sobre todo cuando aún no se cuenta con un instrumento para medir la calidad del servicio de limpieza asociado a la recolección de residuos peligrosos y considerando 
además que este índice subestima la verdadera consistencia de los instrumentos de medición (Maroco, 2006). Como este coeficiente no está indicado para medir la dimensionalidad de una escala aun cuando a simple vista se intuye que está compuesta por más de una dimensión (Oviedo y Campo-Arias, 2005), se tuvo que realizar una análisis factorial para corroborar la composición de las dimensiones e ítems de la escala propuesta.

En el caso de la prueba de esfericidad de Bartlett los grados de libertad fueron de 105 y la significación de $0,000<0,05$, lo que representa una seguridad del $95 \%$, y que la correlación estudiada no ocurre al azar pues cuanto mayores sean los grados de libertad y menor el nivel de significación, más improbable es que la matriz sea una matriz de identidad por lo que es adecuado optar por el análisis factorial. Sin embargo, es importante tener en cuenta que este indicador no pretende sustituir el papel del investigador para evaluar la relevancia de un determinado ítem.

La medida de la adecuación muestral de KaiserMeyer-Olkin tomó un valor de 0,7 , que se encontró dentro del criterio de regular adecuación muestral $(0,6 \leq \mathrm{KMO} \leq 0,7)$ para realizar el análisis factorial. $\mathrm{Si}$ bien un valor igual o superior a 0,80 de la comunalidades de los ítems sugiere que la matriz de correlación será apropiada para factorizar, es poco probable que ocurra en los datos reales ya que en las ciencias sociales las comunalidades van de 0,40 a 0,70 (Velicer y Fava, 1998). En ese sentido Osborne y Costello (2009) mencionan que 0,32 es una buena regla de oro para la carga mínima de un ítem, lo que equivale a aproximadamente el $10 \%$ de la varianza de superposición con los otros ítems de ese factor. Un ítem de "carga cruzada" es un elemento que se carga en los 0,32 o superior en dos o más factores, aspecto que bien puede ser considerado a la hora de decidir la exclusión de un ítem de carga cruzada sobre todo cuando hay varios ítems con cargas fuertes de 0,50 o más en cada factor. Si hay varios ítems con cargas cruzadas estos pueden estar mal redactados o la estructura a priori del factor podría ser defectuosa y cuando un factor tiene menos de tres ítems es generalmente débil e inestable; con cinco o más ítems y cargas de al menos 0,50 son deseables e indican un factor con mayor solidez.

En el proceso de determinar el número de factores a retener en el AFE y dependiendo de las condiciones y técnicas que se utilicen, puede ocurrir una sobreestimación o en el peor de los casos una subestimación del número de factores. La regla Kaiser (valores propios $>$ 1) (K1), es la más popular y consecuentemente la más utilizada. Sin embargo, el uso de esta última demuestra su tendencia a sobreestimar el número de factores subyacentes que deben retenerse hasta en un 1/3, 1/5 y $1 / 6$, el número real de factores. Además su precisión empeora a medida que hay un aumento del número $n$ de variables (si se analizan $n$ variables, el número de factores que obtendrá esta regla oscilará entre $\mathrm{n} / 5 \mathrm{y}$ $\mathrm{n} / 3$, sea la escala unidimensional o no), cuando el tamaño de la muestra aumenta, la magnitud de las comunalidades y la proporción de sujetos por variable son bajas (Navarro y Merino, 2010; Merino y Domínguez, 2015; Ferrando y Aguiano-Carrasco). Sin embargo, Fabrigar et al. (1999), apoyaron su buen funcionamiento cuando los factores comunes están claramente definidos.

Las comunalidades por cada dimensión fueron relativamente altas, lo que implica que todas las variables están muy bien representadas en el espacio de los factores con excepción de la dimensión participación ciudadana que fue la peor explicada en nuestro modelo ya que sólo fue capaz de reproducir el $44,5 \%$ de su variabilidad original (Tabla 1-ApéndiceA).

Tabla 3. Estimación de las comunalidades por dimensión, partiendo de un valor inicial de 1

\begin{tabular}{ll}
\hline Dimensión & Extracción \\
\hline Capacidad respuesta & 0,632 \\
Confiabilidad & 0,626 \\
Profesionalismo & 0,591 \\
Credibilidad & 0,607 \\
Comunicación & 0,619 \\
Participación & 0,445 \\
Seguridad & 0,743 \\
\hline
\end{tabular}

Método de extracción: Análisis de componentes principales 
Como se observa las dimensiones mejores representadas en la variable atributiva fueron seguridad, capacidad de respuesta y confiabilidad no encontrando valores adecuados en participación ciudadana $(0,4)$. Respecto a ésta última, los ítems 15 y 16 fueron los peores explicados y solo el ítem 21 fue el mejor explicado al ser capaz de reproducir el 0,8de su variabilidad original (Tabla 4).

Tabla 4. Estimación de las comunalidades por ítem con un valor inicial de 1

\begin{tabular}{llll}
\hline Ítems & Extracción & Ítems & Extracción \\
\hline P1 & 0,765 & P13 & 0,75 \\
P2 & 0,827 & P14 & 0,567 \\
P3 & 0,7 & P15 & 0,595 \\
P4 & 0,612 & P16 & 0,484 \\
P5 & 0,691 & P17 & 0,688 \\
P6 & 0,622 & P18 & 0,68 \\
P7 & 0,587 & P19 & 0,683 \\
P8 & 0,734 & P20 & 0,631 \\
P9 & 0,519 & P21 & 0,766 \\
P10 & 0,53 & P22 & 0,547 \\
P11 & 0,674 & P23 & 0,635 \\
P12 & 0,598 & & \\
\hline
\end{tabular}

Método de extracción: Análisis de componentes principales

Luego de analizar los ítems respecto a sus comunalidades, se optó por eliminar las preguntas P7, P9, P10, P14, P15, P16 y P22 por presentar valores menores que 0,60 , después se obtuvo ítems bien representados por su valoración factorial como: P1, P2, P11, P18 y P21 con valores por encima de 0,74 (Tabla 5).

Tabla 5. Estimación depurada de las comunalidades por ítem con un valor inicial de 1

\begin{tabular}{llll}
\hline Items & Extracción & Items & Extracción \\
\hline P1 & 0,765 & P13 & 0,577 \\
P2 & 0,804 & P17 & 0,697 \\
P3 & 0,675 & P18 & 0,742 \\
P4 & 0,686 & P19 & 0,695 \\
P5 & 0,632 & P20 & 0,594 \\
P6 & 0,724 & P21 & 0,797 \\
P8 & 0,719 & P23 & 0,604 \\
P11 & 0,747 & & \\
\hline
\end{tabular}

Método de extracción: Análisis de componentes principales

En la determinación del número de factores se encontró que seis factores subyacentes (componentes principales) extraídos explicaron el 69,711\% de la varianza explicada. En la Tabla 6 se observa que el componente seis cumple con la regla de Kaiser con valores autoasociados mayores que uno y con un porcentaje de varianza explicada mayor al $66 \%$.

Son seis los factores que permiten medir la calidad del servicio de limpieza municipal a través de la percepción de los usuarios, los cuales en su mayoría no siguen el planteamiento de los ítems propuestos por cada una de las siete dimensiones que formaron parte de la escala inicial.

La solución factorial sin rotar con autovalores superiores a la unidad mediante la extracción de factores por el método de componentes principales se muestra en la Figura 1.

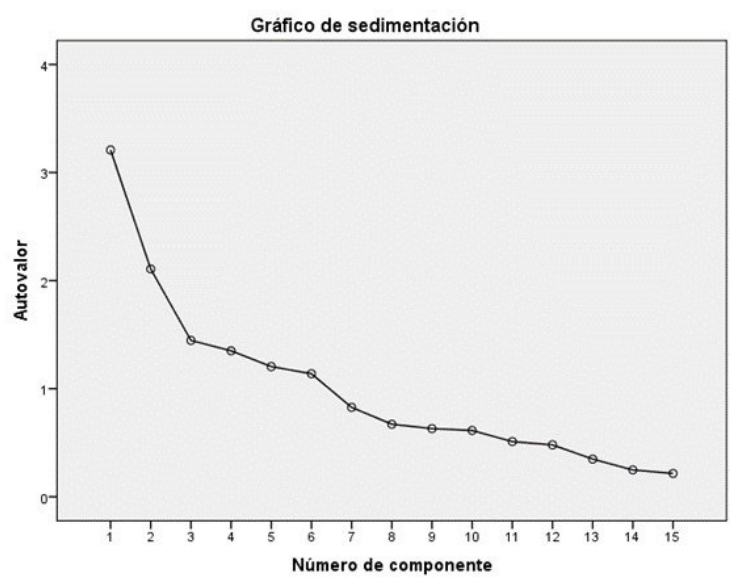

Figura 1. Gráfico de sedimentación.

Se aprecia que los componentes que explicaron el $69,711 \%$ de la variabilidad original de los datos fueron en total seis, lo cual se visualiza en el gráfico de sedimentación de los autovalores, pues a partir del séptimo factor el descenso se estabilizó, lo que significa que los demás componentes tienen una muy baja explicación acerca de la variación de los datos.

Respecto a la variable participación ciudadana y su baja reproducción de la variabilidad original consideramos que puede deberse a que para el usuario no es determinante su participación o colaboración para hacer un juicio cuando se requiere medir y evaluar la calidad de un servicio o que las preguntas mencionadas en la dimensión no fueron las más apropiadas para su medición.

La Tabla 7, denominada matriz de cargas o de saturaciones factoriales, muestra la carga de cada ítem 
en cada componente principal, de forma que los componentes con pesos factoriales más elevados en términos absolutos indican una relación estrecha con los ítems.

El factor uno sustenta el 21,4\% de la varianza acumulada e incluye a la totalidad de la dimensión capacidad de respuesta (ítems 1, 2 y 3) y a los ítems 8, 11 y 13 pertenecientes a las dimensiones de profesionalismo, credibilidad y comunicación, con cargas factoriales de $0,8,0,7,0,6,0,6,0,5$ y 0,6 , respectivamente. A este factor se le denominará nuevamente capacidad de respuesta.

El factor dos justifica el $14,0 \%$ de la varianza acumulada, y comprende a los ítems 19, 20 y 21 de la dimensión de participación ciudadana y el ítem 22 de la dimensión de seguridad, con cargas factoriales de $0,6,0,6,0,6$ y 0,5 , respectivamente. Debido a que el ítem 22 tiene una menor carga se procedió a su reubicación, por lo que este factor mantuvo su denominación de participación ciudadana.

El tercer factor comprende al ítem 4 que explica el $9,7 \%$ de la varianza explicada con una carga factorial de 0,6 que pertenece a la dimensión de confiabilidad.

El factor cuatro explica el 9,0\% de la varianza total y está asociado al ítem 18 , que pertenece a participación ciudadana con una carga de 0,6 . Por tanto consideramos que debe ser fusionado con el factor dos.

El quinto factor explica el $8,0 \%$ de la varianza explicada y está asociado a los ítems 5 (confiabilidad) y 17 (participación ciudadana), con cargas de 0,6 y 0,6, respectivamente. Ambos ítems fueron reubicados en sus dimensiones correspondientes.

El factor seis explica el 7,6\% de la varianza y comprende al ítem 6 de la dimensión confiabilidad con una carga de 0,6 , por lo que se reubicó en el factor tres.

Si bien el instrumento con que se inició el estudio contenía siete dimensiones y un total de 23 ítems y después de la rotación de factores se obtuvo que seis “agrupamientos" con un total de 15 ítems explicaron el $69,7 \%$ de la varianza explicada, se observa que los factores tercero al sexto sólo están constituidos en su mayoría por un solo ítem, lo que indica que las dimensiones profesionalismo, credibilidad, comunicación y seguridad han quedado dispersas, no obstante a estar presentes. Es importante destacar que las comunalidades de los 15 ítems son mayores a 0,5 con lo cual se afirma que las variables originales quedan muy bien explicadas a través de los componentes extraídos.

Al reestructurar la escala se obtuvieron tres dimensiones (factores críticos en nuestra investigación) tales como: capacidad de respuesta, que incrementó tres ítems y quedó con los ítems 1, 2, 3, 8, 11 y 13; luego participación ciudadana, al que se redujo dos ítems y se configuró con los ítems 17, 18, 19, 20 y 21; y finalmente, confiabilidad que incluyó un ítem adicional quedando con los ítems 4, 5, 6 y 23.

Las dimensiones propuestas inicialmente como profesionalismo del personal recolector con tres ítems, credibilidad con dos ítems y comunicación con tres ítems fueron reducidas. Sin embargo, consideramos que se puede mejorar su construcción teórica e incluir en nuevas escalas que desarrollen y estudien su validez para poder contar con resultados definitivos.

Los reajustes a efectos de lograr contar con una escala de medición adecuada pueden pasar por distintos enfoques, desde mantener en cada dimensión los ítems que tienen mayor carga factorial hasta reagrupar los ítems y renombrar las dimensiones, lo cual depende del investigador ya que en definitiva la validez de la escala depende de la asunción cualitativa teórica asumida. Para nuestro caso se optó por considerar al menos tres ítems por dimensión (Streiner, 1994; Fabrigar et al., 1999; Osborne y Costello, 2009; Ferrando y Anguiano-Carrasco, 2010), cumpliendo con el requisito que haya por lo menos tres marcadores por factor si los factores no están correlacionados, y como mínimo dos marcadores por factor si los factores están correlacionados, ya que un solo ítem por dimensión puede generar mediciones poco confiables por el elevado error producto del azar, siendo improbable que abarque la amplitud de una dimensión porque es incapaz de mostrar los diferentes grados o matices, lo cual sería un error de validez de contenido desde el 
enfoque clásico de los test (Streiner, 1994; Jaju y Crask, 1999).

Los desafíos futuros para medir la calidad del servicio de limpieza pública tendrán que seguir la tendencia a realizar mediciones que traspasen las relaciones lineales clásicas y los supuestos de certidumbre e independencia (la respuesta a un ítem no puede estar condicionada a la respuesta dada a otros ítems), profundizando en los efectos curvilíneos relacionales entre constructos y efectos moderadores, el uso de modelos de ecuaciones estructurales exploratorias para estudiar las relaciones de causalidad, pasando por los procesos de análisis jerárquico, análisis factorial confirmatorio, técnicas de análisis de sentimiento, desarrollo de modelos multidimensionales jerárquicos, investigaciones respecto a la incertidumbre de los términos lingüísticos, mediciones de expectativas y percepciones en momentos diferentes, estudios longitudinales así como explorar otras formas de evaluación y profundizar en las particularidades de contextos específicos, lo que implica desarrollar conceptos especiales o profundizar en las funciones del servicio o las dimensiones de calidad más relevantes como las características socioeconómicas y demográficas hasta desarrollar nuevas medidas de la calidad ( Pérez y Medrano, 2010; Cosialls et al., 1992; Silva-Zamora y Schiattino-Lemus, 2008).

\section{CONCLUSIONES}

Los factores críticos asociados al riesgo de recolección de residuos peligrosos por el servicio de limpieza municipal de la ciudad de Chachapoyas son tres: capacidad de respuesta, participación ciudadana y confiabilidad

Con el instrumento inicialmente propuesto en base al análisis de componentes principales, se obtuvo que los factores críticos asociados al riesgo de recolección de residuos peligrosos son seguridad (74,3\%), capacidad de respuesta $(63,2 \%)$ y confiabilidad $(62,6 \%)$. Por otra parte, los que menos lo explicaron fueron profesionalismo $(59,1 \%)$ y participación ciudadana, que es el peor explicado en el modelo pues sólo es capaz de reproducir el 44,5\% de su variabilidad original.

Al aplicar la escala a una muestra de usuarios, la data obtenida permitió confirmar la consistencia del citado instrumento para estimar de manera adecuada el atributo que desea medirse siendo detectadas ciertas debilidades en el mismo lo que sugiere un rediseño de la escala planteada inicialmente, la cual debe ser aplicada a otros usuarios con la finalidad de confirmar los resultados obtenidos.

Como a través del AFE de la escala estudiada sólo se ha realizado el análisis de datos (pues no está diseñado para probar hipótesis o teorías), se recomienda continuar mejorando la "teoría" que sustenta la escala de medición a través del análisis factorial exploratorio para determinar el número de factores subyacentes $\mathrm{y}$ su correspondencia con los ítems. Luego se debe emplear el análisis factorial confirmatorio para aceptar la estructura hipotetizada determinando qué ítems miden qué factores subyacentes, cuáles factores subyacentes están relacionados entre sí, y qué ítems están relacionados con determinados factores subyacentes.

Se requiere continuar investigando para rediseñar el instrumento de medición, de tal forma que se logre plasmar el verdadero impacto en la dimensión participación ciudadana. Así, también se recomienda redefinir otras dimensiones, y según los resultados obtenidos, para ello se debe realizar un nuevo levantamiento de información y realizar análisis factoriales hasta obtener una estructura que satisfaga al investigador y sea representativa de la calidad del servicio. 


\section{APÉNDICE A}

Tabla 1. Escala inicial para medir la calidad del servicio de limpieza municipal

\begin{tabular}{|c|c|c|}
\hline Dimensión & & Ítems \\
\hline \multirow[t]{3}{*}{ Capacidad de respuesta } & 1 & $\begin{array}{l}\text { Los empleados de la municipalidad están disponibles para } \\
\text { atender a la comunida d cuando realiza un reclamo. }\end{array}$ \\
\hline & 2 & $\begin{array}{l}\text { Los empleados de la municipalidad demuestran disposición a } \\
\text { ayudar a la comunidad cuando ésta realiza un reclamo. }\end{array}$ \\
\hline & 3 & $\begin{array}{l}\text { La municipalidad atiende de manera rápida y oportuna las } \\
\text { denuncias y/o necesidades presentadas por la comu nidad. }\end{array}$ \\
\hline \multirow[t]{3}{*}{ Confiabilidad } & 4 & $\begin{array}{l}\text { La municipalidad recoge los desechos sólidos con una } \\
\text { frecuencia diferente a lo que promete. }\end{array}$ \\
\hline & 5 & $\begin{array}{l}\text { La recolección de desechos sólidos se realiza fuera de los días } \\
\text { fijados por la municipalidad. }\end{array}$ \\
\hline & 6 & $\begin{array}{l}\text { La municipalidad no cumple con los hor arios fijados para la } \\
\text { recolección de los desechos sólidos. }\end{array}$ \\
\hline \multirow[t]{3}{*}{ Profesionalismo del personal recolector } & 7 & $\begin{array}{l}\text { Los trabajadores que recogen los desechos sólidos realizan la } \\
\text { recolección de forma cuidadosa. }\end{array}$ \\
\hline & 8 & $\begin{array}{l}\text { El comportamiento de los trabajadores que recogen los } \\
\text { desechos sólidos le inspira confianza. }\end{array}$ \\
\hline & 9 & $\begin{array}{l}\text { Los trabajadores que recogen los desechos sólidos poseen } \\
\text { conocimientos suficientes para responder sus inquietudes } \\
\text { respecto al servicio. }\end{array}$ \\
\hline \multirow[t]{2}{*}{ Credibilidad } & 10 & La municipalidad no cumple con lo que promete a los usuarios . \\
\hline & 11 & $\begin{array}{l}\text { Los trabajadores que recogen los desechos sólidos son } \\
\text { irrespetuosos con los usuarios. }\end{array}$ \\
\hline \multirow[t]{3}{*}{ Comunicación } & 12 & $\begin{array}{l}\text { La municipalidad tiene bien definidos canales de } \\
\text { comunicación con el usuario para recibir reclamos. }\end{array}$ \\
\hline & 13 & $\begin{array}{l}\text { La municipalidad realiza publicidad respe cto a los canales de } \\
\text { comunicación que ofrece al usuario. }\end{array}$ \\
\hline & 14 & $\begin{array}{l}\text { La municipalidad mantiene informado al usuario sobre los días } \\
\text { y horarios de recolección de los desechos sólidos. }\end{array}$ \\
\hline \multirow[t]{8}{*}{ Participación ciudadana } & 15 & $\begin{array}{l}\text { La basura de su vivienda o establecimiento se encuent ra dentro } \\
\text { del inmueble al momento de la recolección de desechos por los } \\
\text { vehículos de la municipalidad. }\end{array}$ \\
\hline & 16 & $\begin{array}{l}\text { Usted conoce las leyes, normas o disposiciones que regulan los } \\
\text { residuos peligrosos. }\end{array}$ \\
\hline & 17 & $\begin{array}{l}\text { Usted desconoce sus deberes y derechos como usuario del } \\
\text { servicio de limpieza pública municipal. }\end{array}$ \\
\hline & 18 & $\begin{array}{l}\text { Usted desconoce formas adecuadas para el manejo de los } \\
\text { desechos generados por su grupo familiar o de trabajo }\end{array}$ \\
\hline & 19 & Usted maneja adecuadamente los residuos peligrosos. \\
\hline & 20 & $\begin{array}{l}\text { Los recipientes en los que almacena los res iduos comunes que } \\
\text { son generados por su familia son adecuados. }\end{array}$ \\
\hline & 21 & $\begin{array}{l}\text { Usted almacena separadamente los residuos peligrosos de los } \\
\text { residuos comunes. }\end{array}$ \\
\hline & & El personal de recolección de desechos sólidos utiliza una \\
\hline \multirow[t]{2}{*}{ Seguridad } & 22 & $\begin{array}{l}\text { vestimenta con la apropiada identifica } \\
\text { municipalidad. }\end{array}$ \\
\hline & 23 & $\begin{array}{l}\text { El personal recolector de desechos sólidos nunca solicita } \\
\text { retribución por el servicio que presta. }\end{array}$ \\
\hline
\end{tabular}


Tabla 2. Matriz de correlaciones entre dimensiones

\begin{tabular}{llccccccc}
\hline & Capacidad & Confiab. & Profes. & Credib. & Comunic & Partic & Seguridad \\
\hline Corr. & $\begin{array}{l}\text { Capacidad } \\
\text { respuesta }\end{array}$ & 1,000 &, 191 &, 219 &,- 181 &, 361 &, 119 &,- 312 \\
& & & & & & & & \\
& Confiabilidad &, 191 & 1,000 &, 101 &, 015 &,- 036 &, 063 &,- 154 \\
& Profesionalismo &, 219 &, 101 & 1,000 &,- 340 &, 318 &, 188 &,- 139 \\
Credibilidad &,- 181 &, 015 &,- 340 & 1,000 &,- 310 &,- 145 &,- 061 \\
Comunicación &, 361 &,- 036 &, 318 &,- 310 & 1,000 &, 199 &,- 055 \\
Participación &, 119 &, 063 &, 188 &,- 145 &, 199 & 1,000 &, 077 \\
& Seguridad &,- 312 &,- 154 &,- 139 &,- 061 &,- 055 &, 077 & 1,000 \\
\hline
\end{tabular}

a. Determinante $=, 484$

Tabla 6. Varianza total explicada

\begin{tabular}{|c|c|c|c|c|c|c|}
\hline \multirow[b]{2}{*}{ Componente } & \multicolumn{3}{|c|}{ Autovalores iniciales } & \multicolumn{3}{|c|}{ Sumas de extracción de cargas al cuadrado } \\
\hline & Total & $\%$ de varianza & $\begin{array}{c}\% \frac{0}{\text { acumulado }}\end{array}$ & Total & $\%$ de varianza & $\begin{array}{c}\% \\
\text { acumulado }\end{array}$ \\
\hline 1 & 3,209 & 21,394 & 21,394 & 3,209 & 21,394 & 21,394 \\
\hline 2 & 2,107 & 14,049 & 35,443 & 2,107 & 14,049 & 35,443 \\
\hline 3 & 1,447 & 9,645 & 45,088 & 1,447 & 9,645 & 45,088 \\
\hline 4 & 1,350 & 9,003 & 54,091 & 1,350 & 9,003 & 54,091 \\
\hline 5 & 1,205 & 8,031 & 62,122 & 1,205 & 8,031 & 62,122 \\
\hline 6 & 1,138 & 7,589 & 69,711 & 1,138 & 7,589 & 69,711 \\
\hline 7 &, 827 & 5,515 & 75,226 & & & \\
\hline 8 &, 671 & 4,473 & 79,699 & & & \\
\hline 9 &, 630 & 4,202 & 83,901 & & & \\
\hline 10 & ,613 & 4,088 & 87,989 & & & \\
\hline 11 &, 510 & 3,402 & 91,391 & & & \\
\hline 12 &, 480 & 3,198 & 94,589 & & & \\
\hline 13 &, 348 & 2,321 & 96,910 & & & \\
\hline 14 &, 248 & 1,653 & 98,563 & & & \\
\hline 15 &, 216 & 1,437 & 100,000 & & & \\
\hline
\end{tabular}

Tabla 7. Matriz de saturaciones

\begin{tabular}{|c|c|c|c|c|c|c|}
\hline \multirow{2}{*}{ Items } & \multicolumn{6}{|c|}{ Componente } \\
\hline & 1 & 2 & 3 & 4 & 5 & 6 \\
\hline P1 &, 774 &,- 296 &, 062 &,- 015 &, 101 &, 252 \\
\hline $\mathrm{P} 2$ & ,693 &,- 504 &, 070 &,- 035 &, 041 & ,249 \\
\hline P3 &, 626 &,- 483 &,- 002 &,- 135 &, 043 &, 171 \\
\hline P4 &, 023 &,- 220 &, 556 & ,088 &, 356 &,- 440 \\
\hline P5 &, 063 &,- 204 &, 371 &, 340 &, 557 &,- 150 \\
\hline P6 &, 334 &,- 125 &, 188 &,- 271 &,- 338 &,- 612 \\
\hline P8 &, 578 & ,194 &,- 255 &, 270 &,- 100 &,- 446 \\
\hline P11 &,- 482 &,- 190 &, 434 &,- 453 & ,093 & ,276 \\
\hline P13 &, 563 & ,009 &,- 303 & ,377 &, 063 & ,148 \\
\hline P17 &, 147 &, 235 & ,461 &, 186 &,- 579 &, 194 \\
\hline P18 &,- 036 &,- 080 &, 490 & ,603 &,- 343 &, 119 \\
\hline P19 &, 513 &, 565 &, 210 &,- 224 &, 131 & ,031 \\
\hline P20 &, 353 &, 582 &, 320 &,- 075 &, 040 &, 145 \\
\hline P21 &, 505 &, 644 &, 094 &,- 300 &, 170 & ,012 \\
\hline P23 &,- 270 &, 469 &,- 055 &, 412 &, 340 &, 152 \\
\hline
\end{tabular}

Método de extracción: análisis de componentes principales. a: 6 componentes extraídos. 


\section{REFERENCIAS BIBLIOGRÁFICAS}

Cosialls, L. S., M. F. Blanxart, y J. G. Olmos. 1992.

“Aplicación del análisis exploratorio de datos en los sistemas de ecuaciones estructurales." Anales de psicología 8 (1-2): 123-130.

DIGESA (Dirección General de Salud Ambiental). 2012. Gestión y Manejo de Residuos Sólidos en Establecimientos de Salud y Servicios Médicos de Apoyo. Norma Técnica de Salud $\mathrm{N}^{\circ}$ 096-MINSA/DIGESA V.01. Ministerio de Salud. Lima (Perú)

Duque, E. J., y C. Ramiro. 2012. "Medición de la percepción de la calidad del servicio de educación por parte de los estudiantes de la UPTC Duitama." Criterio Libre 10 (16): 159192.

Fabrigar, L. R., D. T. Wegener, R. C. MacCallum, y E. J. Strahan. 1999. "Evaluating the use of exploratory factor analysis in psychological re-search." Psychological Methods 4 (3): 272299.

Ferrando, P. J., y C. Anguiano-Carrasco. 2010. “El análisis factorial como técnica de investigación en psicología.” Papeles del Psicólogo 31 (1): 18-33.

Jaju, A., y M. R. Crask. 1999. “The perfect design: Optimization between reliability, validity, redundancy in scale items and response." American Marketing Association 10: 127131.

Kaiser, H. F. 1970. “A second generation Little Jiffy.” Psychometrika 35: 401-415.

Lloret-Segura, S., A. Ferreres-Traver, A. HernándezBaeza, y I. Tomás-Marco. 2014. "El análisis factorial exploratorio de los ítems: una guía práctica, revisada y actualizada.” Anales de psicología 30 (3): 1151-1169.

Maroco, J. 2006. "Qual a fiabilidade do alfa de Cronbach?" Laboratório de Psicologia, 4 (1): 65-90.

Merino, C., y S. Domínguez. 2015. "Sobre la elección del número de factores en estudios psicomé- tricos en la Revista Latinoamericana de Ciencias Sociales, Niñez y Juventud." Revista Latinoamericana de Ciencias Sociales, Niñez y Juventud, 3 (2): 1320-1322.

Navarro, J. S., y C. Merino. 2010. “Un réquiem para la regla de Kaiser (eigen $>1$ ) en la retención del número de factores." Revista de Psicologia, 22 (3): 641-642.

OEFA (Organismo de Evaluación y Fiscalización Ambiental). 2014. Fiscalización ambiental del sector salud a nivel de gobiernos regionales. Índice de cumplimiento de los gobiernos regionales. Informe Técnico. Lima (Perú)

OEFA (Organismo de Evaluación y Fiscalización Ambiental). 2016. Fiscalización ambiental de gestión de sólidos de gestión municipal: Índice de cumplimiento de las municipalidades provinciales a nivel nacional. Informe Técnico. Lima (Perú)

Orjuela-Yepes, D. 2013. "Estudio comparativo de las normas relevantes a nivel internacional para la definición, clasificación, exclusión, desclasificación e identificación de residuos peligrosos." Publicación Científica en Ciencias Biomédicas 11 (9): 73 - 92.

Osborne, J. W., y A. B. Costello. 2009. "Best practices in exploratory factor analysis: Four recommendations for getting the most from your analysis." Pan-Pacific Management Review 12(2): 131-146.

Oviedo, H. C., y A. Campo-Arias. 2005. “Aproximación al uso del coeficiente alfa de Cronbach." Revista Colombiana de Psiquiatría 34 (4): 572-580.

Pérez, E. R., y Medrano, L. 2010. “Análisis Factorial Exploratorio: Bases Conceptuales y Metodológicas." Revista Argentina de Ciencias del Comportamiento 2(1), 58-66.

(PE) Poder Ejecutivo. 2016. Aprueba la ley de gestión integral de residuos sólidos. Decreto Legislativo $\mathrm{N}^{\circ}$ 1278. Lima (Perú)

Sáenz, A. 2011. "Factores críticos para la medición de 
la calidad de servicio del aseo urbano en el municipio Maracaibo.” Revista de Estudios Interdisciplinarios en Ciencias Sociales 13 (3): 329-354.

Silva-Zamora, C., y I. Schiattino-Lemus, I. 2008. Modelos de ecuaciones estructurales ¿qué es eso? Cienc. Trab 10 (29): 106-110.

Streiner, D. L. (1994). "Figuring out factors: the use and misuse of factor analysis." Can J Psychiatry 39: 135-140.

Velicer, W. F., y J. L. Fava, J. L. 1998. "Effects of variable and subject sampling on factor pattern recovery." Psychological Methods $3: 231-251$

Zamora, S., L. Monroy, y C. Chávez. 2009. Análisis factorial: una técnica para evaluar la dimensionalidad de las pruebas. Cuaderno técnico 6 . México D.F. (México): Centro Nacional de Evaluación para la Educación Superior 